\title{
Performance of phase noisy optical systems with frequency stabilization
}

\author{
Pierre A. Humblet and John S. Young * \\ Massachusetts Institute of Technology \\ Laboratory for Information and Decision Systems \\ Cambridge, Massachusetts 02139-4307 \\ Revised January 23, 1992
}

\begin{abstract}
Laser phase noise causes a significant performance degradation of coherent optical communication systems. In this paper we analyze its effect for a model more general than usually considered. We evaluate bounds and approximations for the probability of error of binary orthogonal modulation, such as wide deviation Frequency Shift Keying, paying particular attention to the effects of frequency feedback stabilization on system robustness.
\end{abstract}

\footnotetext{
*The authors gratefully acknowledge support for this research from NSF (grant NCR-8802991), ARO (contract DAAL03-86-K-0171), NEC and DARPA (grant F19628-90-C-0002). J.S. Young is now with Churchill College, Cambridge, England.
} 


\section{Introduction}

Phase noise in diode lasers is a major cause of degradation of the performances of optical coherent communication systems. These phase fluctuations are due to spontaneous photon emissions within the laser cavity [1]. Numerous papers analyze the effect of phase noise when it is modeled as a Wiener process, e.g. the phase noise is the integral of white frequency noise $[2],[3],[4],[5],[6],[7],[8],[9]$. We consider here a more general model. It sheds light on the nature of the analytical techniques used previously. It also allows us to study the influence of frequency stabilization schemes (e.g. $[10,11,12,13,14,15])$ that reduce the low frequency components of frequency noise. Other important phenomena, such as the influence of the laser relaxation oscillations, or the effect of the increased noise at low frequencies, could also be studied by the techniques developed here.

The remainder of this paper is organized in the following manner. In section 2 we introduce the phase noise model. In section 3 we examine the characteristics of the signal spectrum and evaluate the error probability by deriving a lower bound, an upper bound, and an approximation. The key results of the paper are in the section on the upper bound. Finally in section 4 we apply the results to a simple frequency stabilization scheme. In section 5 we give the results and conclusions.

\section{Signal models}

The unmodulated signals have the form $\Re\left(A e^{j\left(2 \pi f_{c} t+\theta(t)\right)}\right)$ where $f_{c}$ is either the optical carrier frequency, or, in the case of coherent reception, the intermediate frequency. In that expression the phase noise $\theta(t)$ is $2 \pi$ times the integral of the frequency noise $w(t) . w(t)$ is often modeled [16] as a white Gaussian noise process with spectral density $\beta / 2 \pi$, so that the unmodulated signal has a Lorentzian lineshape with bandwidth $\beta$. In this work $w(t)$ is still Gaussian, but we allow a general spectral density $S_{w}(f)=\beta S(f) / 2 \pi$. Explicitly keeping the parameter $\beta$ allows us to examine the effect of changing the intensity, but not 
the spectral shape, of the frequency noise. The shape $S(f)$ might be used to model the effect of frequency stabilization ${ }^{1}$, or other phenomena such as the relaxation oscillation.

It will prove convenient to normalize time with respect to the bit duration $T$ and to scale the phase noise process, so we define the process

$$
w^{\prime}(t)=\sqrt{\frac{2 \pi T}{\beta}} w(t T) .
$$

Its spectral density is $S(f / T)$, and it is independent of $\beta$. Having defined $w^{\prime}$, we also define its integral,

$$
\psi(t)=\int_{0}^{t} w^{\prime}(u) d u
$$

with correlation function

$$
K_{\psi}(t, s)=\int_{0}^{t} \int_{0}^{s} K_{w^{\prime}}\left(\tau-\tau^{\prime}\right) d \tau d \tau^{\prime}
$$

From these definitions, the phase noise $\theta(t)$ can be expressed as

$$
\theta(t)=2 \pi \int_{0}^{t} w(u) d u=\sqrt{2 \pi \beta T} \int_{0}^{t / T} w^{\prime}(u) d u=\sqrt{\gamma} \psi(t / T)
$$

where $\gamma$ is $2 \pi \beta T$. From now on we will always express the phase noise process in terms of $\gamma$ and $\psi(t)$.

We consider the heterodyne receiver shown in figure 1 . The signal is a pulse of phase noisy light of duration $\mathrm{T}$, modulated either by On/Off keying or some binary orthogonal modulation, such as Frequency Shift Keying (FSK), Polarization modulation, or Binary Pulse Position Modulation ${ }^{2}$ (FSK is shown in the figure). Following coherent reception, the electrical signals are passed through IF matched filters, with impulse response $\exp \left(j 2 \pi f_{i} t\right) / T, 0<t<T$, where the $f_{i}$ 's are the intermediate frequencies corresponding to the signals. These matched filters reject the strong shot noise introduced by the coherent receiver. The envelopes of the matched filter outputs are sampled and sent to a decision device.

\footnotetext{
${ }^{1}$ In the case coherent receivers, $S(f)$ relates to the difference of the frequency noises of the transmitter and of the local oscillator. Frequency stabilization greatly affects $S(f)$ at low frequencies.

${ }^{2}$ In that case $T$ must be redefined appropriately
} 
The probability of error achieved with this structure is identical to that of quadrature homodyne receivers using matched filters and squaring circuits. The results also apply to a direct detection system using a front end optical matched filter [17] (or filters, in the case of FSK) when spontaneous noise from in-line optical amplifiers dominates.

If the phase noise is large, it is better [18], [4], [19] to use an IF filter with a wider passband than a matched filter, and to insert another stage of filtering after envelope detection. The analysis of such a system under a general phase noise model will not be attempted here.

\section{Performance Analysis}

We wish to find the probability of error for the system described in the previous section. The coherent reception process introduces noise that can be modeled as white and Gaussian, with two-sided spectral density $N_{0} / 2$.

In the case of orthogonal signals ${ }^{3}$ if signal 1 is sent the outputs of the envelope detectors at time $k T$ are

$$
\begin{gathered}
Y_{0}(k)=\left|n_{c 0}+j n_{s 0}\right|^{2} \\
Y_{1}(k)=\left|\frac{A}{2} \frac{1}{T} \int_{(k-1) T}^{k T} e^{j \sqrt{\gamma} \psi(t / T)} d t+n_{c 1}+j n_{s 1}\right|^{2}
\end{gathered}
$$

where $\gamma$ and $\psi(t)$ include the effects of the phase offset between the signal and the local oscillator, $A$ is the signal amplitude at the output of the detector, $n_{c i}$ and $n_{s i}$ are the in-phase and quadrature noise samples at the output of the matched filters. The noise components are zero mean independent Gaussian random variables with variance $N_{0} / 4 T$. The decision is made by comparing $Y_{0}$ and $Y_{1}$.

It is well known [20], [21], [19] that conditioned on the phase noise process the prob-

\footnotetext{
${ }^{3}$ For FSK we assume a wide deviation system so that a signal component appears only at the output of the corresponding filter.
} 
ability of error for the kth bit is given by:

$$
P(\text { error } \mid \psi)=\frac{1}{2} e^{-\zeta X_{k} / 2} \doteq P_{e}\left(X_{k}\right)
$$

where $\zeta$ denotes the signal to noise ratio $\zeta=A^{2} T / 2 N_{0}$ and

$$
\begin{aligned}
X_{k} & =\left|\frac{1}{T} \int_{(k-1) T}^{k T} e^{j \sqrt{\gamma} \psi(t / T)} d t\right|^{2}=\left|\int_{(k-1)}^{k} e^{j \sqrt{\gamma} \psi(t)} d t\right|^{2} \\
& =\int_{k-1}^{k} \int_{k-1}^{k} e^{j \sqrt{\gamma}(\psi(t)-\psi(s))} d t d s \\
& =\int_{k-1}^{k} \int_{k-1}^{k} \cos (\sqrt{\gamma}(\psi(t)-\psi(s))) d t d s .
\end{aligned}
$$

The key problem is to evaluate the average of the conditional error probability over $\psi(t)$, or equivalently over $X_{k}$.

In the case of On/Off keying, there is only one output. Its value is given by $Y_{0}$ above if no signal is present, and by $Y_{1}$ if there is a signal. The value of the output is compared to a threshold $h$ to obtain a decision. The conditional probability of error can be found $[20],[21]$ to be

$$
P(\operatorname{error} \mid \psi)=\frac{1}{2} e^{-h / N_{0}}+\frac{1}{2}\left(1-Q\left(\sqrt{2 \zeta X_{k}}, \sqrt{2 h / N_{0}}\right)\right)
$$

where $Q()$ denotes the Marcum $Q$ function and $X_{k}$, defined above, is again a sufficient statistics to express the effect of the phase noise.

Note that $\psi(t)-\psi(s)=\int_{s}^{t} w^{\prime}(u) d u$ and that the statistics of this quantity are not affected by simultaneous shifts of $s$ and $t$, because $w^{\prime}(t)$ is stationary. Accordingly the statistics of $X_{k}$ are independent of $k$. From now on we consider only $k=1$ and we omit the subscript. This remark also justifies the fact that we have not added an initial value to the integrals in equation (3).

In the remainder of this section we examine the spectrum of the unmodulated signal and use three different methods to bound and approximate the probability of error. To keep the paper short, we will only provide explicit results for orthogonal modulation, those for On/Off keying can be obtained by the same method. 


\subsection{Unmodulated signal spectrum}

The variance $\mathrm{v}(t-s)$ of $\psi(t)-\psi(s)$ turns out to be a key quantity that determines the spectrum of the unmodulated signal. It is easy to see that

$$
\begin{aligned}
\mathrm{v}(t-s) & =K_{\psi}(t, t)+K_{\psi}(s, s)-2 K_{\psi}(t, s) \\
& =\int_{s}^{t} \int_{s}^{t} K_{w^{\prime}}\left(\tau-\tau^{\prime}\right) d \tau d \tau^{\prime} \\
& =\int_{-\infty}^{\infty} S\left(\frac{f}{T}\right)\left(\frac{\sin (\pi f(t-s))}{\pi f}\right)^{2} d f
\end{aligned}
$$

The last equality follows from the second one by expressing $K_{w^{\prime}}$ in terms of $S(f)$ and then integrating.

The low-pass envelope $K_{s}(\tau)$ of the correlation function of the unmodulated signal of unit power can be written as

$$
\begin{aligned}
K_{s}(\tau) & =E \cos (\theta(t+\tau)-\theta(t)) \\
& =\exp \left(-.5 \gamma \mathrm{v}\left(\frac{\tau}{T}\right)\right)
\end{aligned}
$$

where the second equality stems from the characteristics function of the Gaussian random variable $\theta(t+\tau)-\theta(t)$ and from the relation (3) between $\theta$ and $\psi$. We can immediately draw some conclusions about the relationship between $S(f)$ and the linewidth of the unmodulated signal.

First, when the frequency noise is white, $S(f)=1, \mathrm{v}(\tau)=|\tau|$ and $K_{s}(\tau)=\exp (-\pi \beta|\tau|)$. Its Fourier transform is the familiar Lorentzian $2 \pi \beta /\left((\pi \beta)^{2}+(2 \pi f)^{2}\right)$.

Second, the $\sin (\pi(t-s) f)^{2}$ in (10) can be expressed as $.5(1-\cos (2 \pi(t-s) f))$. For large $|t-s|$, the $\cos ()$ term will bring a negligible contribution to the integral and the limit of $v(\tau)$ as $\tau$ grows (if it exists) will be given by

$$
v(\infty)=\frac{1}{2 \pi^{2} T} \int_{-\infty}^{\infty} \frac{S\left(f^{\prime}\right)}{f^{\prime 2}} d f^{\prime}
$$

where we have made the change of variable $f^{\prime}=f / T$. For the right hand side to be finite there must be an $\epsilon>0$ such that $S(f)$ increases more slowly than $|f|^{1-\epsilon}$ for large $|f|$, 
and more slowly than $|f|^{1+\epsilon}$ for small $|f|$ (the second condition implies that $S(0)=0$, i.e. there is no d.c. frequency error).

If $\mathrm{v}(\infty)$ exists and is finite, $K_{s}(\tau)$ in turn converges to $\exp \left(-\beta /(2 \pi) \int_{-\infty}^{\infty} S(f) / f^{2} d f\right)$ when $\tau$ grows, and this quantity is strictly positive. It corresponds to the amplitude of a discrete "d.c. line" in the spectral density of the of the low pass envelope of the correlation function of the unmodulated signal, as it is the Fourier transform of $K_{s}(\tau)$.

\subsection{Lowerbound on the Probability of Error}

$P_{e}(X)$, defined in (4), is a convex $\cup$ function of $X$ in the interval $[0,1]$, therefore by Jensen's inequality the probability of error is bounded below by

$$
P_{e} \geq \frac{1}{2} e^{-\zeta \bar{X} / 2}
$$

$\bar{X}$ can be found from the correlation function of the phase noise process as follows. From

$$
\bar{X}=\int_{0}^{1} \int_{0}^{1} E\left[e^{j \sqrt{\gamma}[\psi(t)-\psi(s)]}\right] d t d s .
$$

$\psi(t)-\psi(s)$ is a zero mean Gaussian random variable with variance $\mathrm{v}(t-s)$ defined above, so $\bar{X}$ is just the integral of the characteristic function of that random variable evaluated at $\sqrt{\gamma}$ :

$$
\bar{X}=\int_{0}^{1} \int_{0}^{1} e^{-.5 \gamma \mathbf{v}(t-s)} d t d s
$$

\subsection{Upperbound on the Probability of error}

Foschini and his coworkers [6] derived a lower bound $X_{L}$ on $X$ by applying the inequality $\cos (x) \geq 1-x^{2} / 2$ to $(6)$. A quantity closely related to $X_{L}$ was originally introduced in $[5]^{4}$. Both are first order approximations in $\gamma$, and we refer to $X_{L}$ as the linear approximation. They obtain:

$$
X \geq X_{L}=1-\frac{1}{2} \gamma \int_{0}^{1} \int_{0}^{1}(\psi(t)-\psi(s))^{2} d t d s
$$

\footnotetext{
${ }^{4}$ Our $X$ corresponds to $X^{2}$ in [4], [5] and [6].
} 


$$
\begin{aligned}
& =1-\gamma \int_{0}^{1}\left(\psi(t)-\int_{0}^{1} \psi(u) d u\right)^{2} d t \\
& =1-\gamma \int_{0}^{1} y^{2}(t) d t
\end{aligned}
$$

where

$$
y(t)=\psi(t)-\int_{0}^{1} \psi(u) d u
$$

$P_{e}(X) \leq P_{e}\left(X_{L}\right), P_{e} \leq E_{y}\left(P_{e}\left(X_{L}\right)\right)$, and our goal is to evaluate that last quantity. The process $y(t)$ is key to our analysis. It has a correlation function

$$
\begin{aligned}
K_{y}(t, s) & =E\left[\left(\psi(t)-\int_{0}^{1} \psi(u) d u\right)\left(\psi(s)-\int_{0}^{1} \psi(v) d v\right)\right] \\
& =K_{\psi}(t, s)-\int_{0}^{1} K_{\psi}(s, u) d u-\int_{0}^{1} K_{\psi}(t, v) d v+\int_{0}^{1} \int_{0}^{1} K_{\psi}(u, v) d u d v
\end{aligned}
$$

To obtain the statistics of $X_{L}$, we expand the process $y(t)$ in a Karhunen-Loève series $[20]$ on $[0,1]$

$$
y(t)=\sum_{i=1}^{\infty} y_{i} \phi_{i}(t), t \in[0,1]
$$

where the $\phi_{i}$ 's are orthonormal on $[0,1]$ and the $y_{i}$ 's are independent Gaussian random variables with zero mean and variance $\lambda_{i} . \lambda_{i}$ and $\phi_{i}$ satisfy the integral equation

$$
\int_{0}^{1} K_{y}(t, s) \phi_{i}(s) d s=\lambda_{i} \phi_{i}(t), t \in[0,1]
$$

Introducing (15) in (13) and using the orthonormality property yields

$$
X_{L}=1-\gamma \sum_{i=1}^{\infty} y_{i}^{2}
$$

Thus $X_{L}$ is a sum of squares of independent Gaussian variables, and it is completely specified by the variances $\lambda_{i}$. We will return to it later, but focus first on the integral equation (16).

This eigenvalue equation is somewhat formidable, as $K_{y}$ is itself expressed in (14) as a sum of four terms involving $K_{\psi}$. The equation can be simplified by noticing that $\int_{0}^{1} y(t) d t=0$. It follows that $\int_{0}^{1} K_{y}(t, s) d t$ is 0 for all $s$ and that the constant function is 
a solution of the equation, with 0 eigenvalue. This makes physical sense, as a constant phase offset does not affect $X$. The other eigenfunctions are orthogonal to that constant eigenfunction, and their integral over $[0,1]$ is 0 . Consequently the last two terms in (14), which do not depend on $s$, play no role in the eigenvalue equation for the non-constant eigenfunctions. Therefore the integral equation simplifies to

$$
\int_{0}^{1} K_{\psi}(t, s) \phi(s) d s-\int_{0}^{1} \int_{0}^{1} K_{\psi}(s, u) \phi(s) d s d u=\lambda \phi(t), t \in[0,1] .
$$

The integral over $t$ of the left hand side is 0 , which implies that solutions to the simplified equation with non zero eigenvalues also have a 0 integral. Thus they are solutions of the original Karhunen-Loève equation, and we have not introduced extra solutions by omitting terms.

The second term in the left hand side of (18) is independent of $t$. Thus if one differentiates both sides with respect to $t$ one obtains the same differential equation as for the expansion of $\psi(t)$; only the boundary conditions are different. For example in the case of white frequency noise where $K_{\psi}(t, s)=\min (t, s)$, the cosine expansion used in [4] without explicit reference to Karhunen-Loève theory, actually solves the eigenvalue equation (18). It also solves the differential equation (but not the integral equation) associated with the eigenfunctions of the Wiener process [20].

It is also worth noting that the terms in $K_{\psi}$ (if any) that are constant, or functions of $s$ only, or $t$ only, make no contribution to (18). For example one can write $\min (t, s)=$ $.5(s+t-|t-s|)$, and equation (18) with $K_{\psi}=-.5|t-s|$ yields the same answer as with the correlation function $\min (t, s)$ of the Wiener process.

We now return to examining $X_{L}$ under the form (17). Its mean follows easily:

$$
\begin{aligned}
\overline{X_{L}} & =1-\gamma \sum_{i=1}^{\infty} \lambda_{i} \\
& =1-\gamma\left(\int_{0}^{1} K_{\psi}(t, t) d t-\int_{0}^{1} \int_{0}^{1} K_{\psi}(t, s) d t d s\right)
\end{aligned}
$$

The second equality can be obtained directly from (13), or by using a theorem [20] about 
the sum of the eigenvalues.

It is convenient to define $\Psi=\sum_{i=1}^{\infty} y_{i}^{2}$. Because $\Psi$ is a sum of squares of independent Gaussian variables, its density function $p_{\Psi}(x)$ is a convolution of an infinite number of independent chi-squared distributions, with characteristic function

$$
P_{\Psi}(v)=E\left(e^{j v \Psi}\right)=\prod_{i=1}^{\infty} \frac{1}{\sqrt{1-j 2 v \lambda_{i}}}
$$

The tail of the $p_{\Psi}(x)$ will be dominated by the largest eigenvalue, say $\lambda_{1}$, and will behave like $\exp \left(-x / 2 \lambda_{1}\right)$ for large $x$ [21]. The tilted density $p_{\Psi}(x) \exp \left(\alpha x / 2 \lambda_{1}\right)$, where $\alpha$ is a well chosen number such as .8 , can be obtained without numerical difficulty from $P_{\Psi}(v)$ by Inverse Fourier Transform routines.

Now that we have the density for $\Psi$, the probability of error can be bounded. For FSK the probability of error is

$$
\begin{aligned}
P_{e} & \leq E_{\Psi}[.5 \exp (-.5 \zeta(1-\gamma \Psi))] \\
& =.5 \exp (-.5 \zeta) P_{\Psi}(-j \zeta \gamma / 2) \\
& =.5 e^{-.5 \zeta} \prod_{i=1}^{\infty} \frac{1}{\sqrt{1-\zeta \gamma \lambda_{i}}}
\end{aligned}
$$

This expression first decreases with increasing $\zeta$, but it eventually increases without bound as $\zeta$ approaches $1 / \gamma \lambda_{1}$. This is because $X_{L}$ can be negative, even though $X$ lies in $[0,1]$.

A better upperbound is obtained by truncating $X_{L}$ at 0 , yielding

$$
P_{e} \leq .5 \operatorname{Pr}(\Psi>1 / \gamma)+.5 e^{-.5 \zeta} \int_{0}^{1 / \gamma} p_{\psi}(x) e^{.5 \zeta \gamma x} d x
$$

The second term decreases with increasing $\zeta$, but the first term is independent of $\zeta$. Because $\Psi>y_{1}^{2}$, that floor is at least as large as (but usually close to) $2 Q\left(1 / \sqrt{\gamma \lambda_{1}}\right.$ ), where $Q(x)$ denotes that the probability that a zero mean, unit variance Gaussian random variable exceeds $x$. 


\subsection{Exponential approximation}

Although it has the virtue to provide a bound, the approximation $X_{L}=1-\gamma \Psi$ is inaccurate for large $\gamma$ as it can become negative although the original $X$ lies in $[0,1]$. To remedy this Azizoglu and Humblet [19] have suggested using the exponential approximation

$$
X_{E}=e^{-\gamma \Psi}
$$

which always lies in the desired interval and is also a first order approximation in $\gamma$. For the case of the simple Brownian motion phase noise there is some justification for this. The first, second and fourth moments of $X_{E}$ are known to be in very close agreement with the actual moments of $X$ for all $\gamma$. This is better than the moments of the bound $X_{L}$ which only agree in the region of small $\gamma$. However the moments provide little information about the correctness of the approximate density for small values of $X$, although those values can greatly influence the error probability.

The mean of $X_{E}$ is simply given by

$$
\overline{X_{E}}=P_{\psi}(j \gamma)=\prod_{i=1}^{\infty} \frac{1}{\sqrt{1+2 \gamma \lambda_{i}}}
$$

which can be compared with the mean of $X$ given in (12) to gain confidence in the exponential approximation.

The probability of error can be evaluated using the previously calculated statistics for $\Psi$.

$$
\begin{aligned}
P_{e} & \approx E_{X_{E}}\left[.5 e^{-.5 \zeta X_{E}}\right] \\
& =E_{\Psi}\left[.5 \exp \left(-.5 \zeta e^{\gamma \Psi}\right)\right]
\end{aligned}
$$

\section{Frequency Feedback Stabilization}

Our interest in frequency feedback stabilization stems from a number of reasons. The fact that the white frequency noise model results in plausible performance, despite having 
infinite mean "frequency power", suggests that the low frequency components of the frequency noise most degrade the performance, and that it would be beneficial to reduce them. This view is reinforced by considering the variance derived in (10). On a more practical level, in heterodyne systems the local oscillator frequency tracks the transmitter frequency, matching it best when fluctuations are slow. This leads to a non white $S(f)$. Also, a number of recent experiments have successfully reduced the frequency noise by using feedback. The usual method (e.g. $[10,11,12,13,14,15])$ is to pass part of the laser output through a frequency discriminator, consisting of an optical filter with a steep transmissivity slope in the nominal frequency region, followed by a photodetector. The output of the photodetector is filtered and then used to provide electronic feedback to the laser driving circuits. A diagram of this system, as well as a traditional model of the linearized frequency loop, appear in fig. 2 .

For the sake of mathematical convenience we assume that the open loop frequency noise spectral density is $S_{n}(f)=\beta / 2 \pi$ (i.e. the usual white noise model), and that the loop filter is a simple integrator with gain $b$ and delay $d$ so that $H(f)=b \exp (-j 2 \pi f d) /(j 2 \pi f)$. The closed loop system is stable if and only if $b d<\pi / 2$. The closed loop output has a spectral density given by

$$
S_{n}(f)\left|\frac{1}{1+H(f)}\right|^{2}=\frac{\beta}{2 \pi} \frac{(2 \pi f)^{2}}{(2 \pi f)^{2}-4 \pi f b \sin (2 \pi f d)+b^{2}}
$$

If $b d \ll \pi / 2$ the delay $d$ can be neglected and we have a first order loop with a $3 \mathrm{~dB}$ cutoff frequency $b / 2 \pi \ll .25 / d$. We assume this situation in the rest of the paper. It is about the simplest example of a system that tracks low frequencies very well, but cannot follow high frequencies. Typically this is due to limits on the electronics, or on the laser frequency response, or on delays in the feedback loop. In practice, these factors will determine the cutoff frequency.

From the definition and formulas in section 2 the normalized frequency noise $w^{\prime}(t)$ 
has a spectral density

$$
S_{w^{\prime}}(f)=\frac{(2 \pi f)^{2}}{(2 \pi f)^{2}+(b T)^{2}} .
$$

We will find it convenient to define the parameter $r=b T$. The dimensionless quantity $r / 2 \pi$ expresses the $3 \mathrm{~dB}$ cutoff of the frequency noise in terms of the baud rate.

By inverting $S_{w^{\prime}}(f)$ one finds the correlation function of $w^{\prime}$ to be

$$
K_{w^{\prime}}(\tau)=\delta(\tau)-.5 r e^{-r|\tau|}
$$

From (2) the correlation function of the normalized phase noise process $\psi(t)$ is,

$$
K_{\psi}(t, s)=\frac{1}{2 r}\left(1+e^{-r|t-s|}-e^{-r t}-e^{-r s}\right) .
$$

From (8) the variance of $\psi(t+\tau)-\psi(\tau)$ is $\mathrm{v}(\tau)=\left(1-e^{-r|\tau|}\right) / r$. The envelope of the correlation function of the unmodulated signal is given by (11):

$$
K_{s}(\tau)=\exp \left(-\frac{\gamma\left(1-e^{-r|\tau| / T}\right)}{2 r}\right)=\exp \left(-\frac{\pi \beta\left(1-e^{-b|\tau|}\right)}{b}\right)
$$

and its Fourier transform is

$$
\begin{aligned}
S_{s}(f) & =\exp \left(-\frac{\pi \beta}{b}\right) \delta(f)+\int_{-\infty}^{\infty}\left(\exp \left(-\frac{\pi \beta\left(1-e^{-b|\tau|}\right)}{b}\right)-\exp \left(-\frac{\pi \beta}{b}\right)\right) \exp (-j 2 \pi f \tau) d \tau \\
& =\exp \left(-\frac{\pi \beta}{b}\right)\left(\delta(f)+\int_{-\infty}^{\infty}\left(\exp \left(\frac{\pi \beta e^{-b|\tau|}}{b}\right)-1\right) \exp (-j 2 \pi f \tau) d \tau\right) \\
& \approx\left(1-\frac{\pi \beta}{b}\right)\left(\delta(f)+\frac{2 \pi \beta}{b^{2}+(2 \pi f)^{2}}\right), \quad b>>\beta
\end{aligned}
$$

As we have seen, in absence of feedback this spectrum is a Lorentzian of width $\beta$. As $b$ increases, a discrete d.c. line of power $\exp (-\pi \beta / b)$ appears, with the rest of the power distributed in a continuous spectrum of increasing width. When $b$ is much larger than $\beta$, the continuous part approaches a Lorentzian of width $b / \pi$. The d.c. line remains when the loop delay is significant, but the continuous part has a more complicated structure. 


\subsection{Lower Bound}

To use Jensen's inequality we use the variance $\mathrm{v}(\tau)$ in $(12)$ to find the mean of $X$ :

$$
\bar{X}=\int_{s=0}^{1} \int_{t=s}^{1} \exp \left(-\frac{.5 \gamma}{r}\left(1-e^{-r|t-s|}\right)\right) d t d s .
$$

This is not available in closed form but can be evaluated numerically. Finally using $P_{e} \geq .5 e^{-.5 \zeta \bar{X}}$ we get a lowerbound for the received probability of error as a function of $\zeta$ for different values of $r$ and $\gamma$. The resulting bounds for $\gamma=2$ and selected values of $r$ appear in fig. 3. Note that for the sake of clarity not all the figures of the paper show the same values of $r$, but that the line types for the values of $r$ are the same in all the figures.

\subsection{Upper Bound}

To obtain the upperbound we must solve the key equation (18). $K_{\psi}$ was obtained before, and according to the remark in section 3.3 , only the $\exp (-r|t-s|) / 2 r$ term is significant. Introducing it in (18) and differentiating twice with respect to $t$ leads to a simple equation in terms of $\phi(t)$ and $\lambda$ :

$$
\frac{d^{2} \phi(t)}{d t^{2}}=\left(r^{2}-\frac{1}{\lambda}\right) \phi(t)-\frac{r}{\lambda} \int_{0}^{1} \int_{0}^{1} e^{-r|u-s|} \phi(s) d s d u .
$$

If $r=0$ the right-most term disappears.

The general solution is $\phi(t)=A+B \sin (\alpha t)+C \cos (\alpha t)$, with $\alpha=\sqrt{1 / \lambda-r^{2}}$. It satisfies the integral equation only for a countable set of values $\alpha_{i}, i=1,2,3 \ldots$, with corresponding eigenvalues

$$
\lambda_{i}=\frac{1}{\alpha_{i}^{2}+r^{2}} .
$$

The algebra to find these values is greatly simplified by noting that $\phi(t)$ must have either odd or even symmetry about the point $t=.5^{5}$. For the odd symmetry case $\alpha$ must satisfy

\footnotetext{
${ }^{5}$ Working on the interval $[-.5, .5]$ rather than $[0,1]$ also helps.
} 
$\alpha \cot (\alpha / 2)=-r$. For small $r$, the solutions have the form $\alpha_{i}=i \pi+2 r / i \pi, i=1,3,5, \ldots$ and they approach $(i+1) \pi$ as $r$ grows.

In the case of even symmetry, the equation is $\tan (\alpha / 2)=r^{2} \alpha /\left(2 r^{2}+\alpha^{2}(2+r)\right)$. For small $r$, the solutions are $\alpha_{i}=i \pi+r^{2} / i \pi, i=2,4,6, \ldots$ As $r$ approaches infinity, the equation becomes $\tan (\alpha / 2)=\alpha / 2$ so that $\alpha_{i}$ remains below $(i+1) \pi$. Thus for both the odd and even cases $r$ does not affect $\alpha_{i}$ by more than $\pi$.

If $r=0$ the previous theory leads to Foschini's cosine expansion with eigenvalues $\lambda_{i}=1 /(\pi i)^{2}$. For small $r>0$, the eigenvalues are $\lambda_{i} \approx 1 /\left((i \pi)^{2}+4 r\right), i=1,3, \ldots$ and $\lambda_{i} \approx 1 /\left((i \pi)^{2}+3 r^{2}\right), i=2,4, \ldots$. Thus the odd eigenvalues are more affected by $r$. We show in fig. 4 the first two eigenvalues as functions of $r$.

To give an intuitive feel for the effect of frequency feedback on $\Psi$, we show in fig. 5 a plot of the density function of $\Psi$ for different values of $r$. Note that the slope of the tail in figure 5 is about $1 /\left(2 \lambda_{1} \ln (10)\right)$. The first 240 eigenvalues were used in all our calculations.

The upperbound on $P_{e}$ obtained from (21) appears in fig. 6,7 and 8 for $\gamma=.2,1$ and 2 respectively.

\subsection{Exponential Approximation}

To gain confidence in the approximation $X_{E}$ we compare in fig. 9 the means $\bar{X}, \overline{X_{L}}$ and $\overline{X_{E}}$ obtained from formulas $(25),(19)^{6}$ and (22).

The approximate values of $P_{e}$ obtained from (23) appear in fig. 10 and 11 for $\gamma=1$ and 2 .

\section{Results and Conclusions}

Comparing figures 3,8 and 11 , or examining the summary in fig. 12 shows that there is a big gap between the bounds for large $\gamma$ and small values of $r$. The exponential

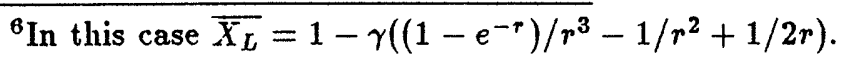


approximation falls neatly in the middle. We do not know where the exact expression lies. To find out one might use a numerical technique as in [8], although it is likely to be cumbersome for general phase noises.

Yet, one would normally never operate in a region far from the "no phase noise" curve, as there are receiver structures [18], [4], [19] which permit operation with less than about $1 \mathrm{~dB}$ penalty for $P_{e}=10^{-9}$ and $\gamma=2$. For values of $r$ large enough for the penalty to be small, all the bounds and approximations are in excellent agreement. One sees from figures 6,7 and 8 that such operation occurs for $(\gamma=.2, r=0),(\gamma=1, r=5)$ and $(\gamma=2, r=10)$. This can be explained with the help of fig. 4 showing the largest eigenvalues. In these three cases the product of $\gamma$ and $\lambda_{1}$ is about .02 , and it is this product that determines the tail of the distribution of $X_{L}$.

We can thus conclude that having the $3 \mathrm{~dB}$ frequency cutoff of the frequency noise spectral density about equal to the bit rate $(r=2 \pi)$ allows the simple receiver considered here to tolerate $\gamma \approx 1$. This represents a five fold increase in $\gamma$ over the case without frequency stabilization. Extending the cutoff to twice the bit rate allows a ten fold increase. For even larger $r$ one should take into account the higher order eigenvalues as they become significant compared to $\lambda_{1}$, and eventually pay more attention to the model of the frequency noise at high frequencies. It is important to note that the improvement due to frequency feedback is much less than might be expected on the basis of the spectral density derived in (24). Common definitions of linewidth may not be very significant for frequency stabilization systems in communication applications.

Acknowledgements Many thanks are due to Murat Azizoglu, now with George Washington University, for his help and encouragement during the course of this research. He and Rick Barry also carefully read the paper and provided thoughtful comments. 


\section{References}

[1] C. H. Henry, "Theory of linewidth of semiconductor lasers," IEEE J. Quantum Electron., vol. QE-18, pp. 259-264, Feb. 1982.

[2] J. Salz, "Coherent lightwave communications," AT\& T Technical Journal, vol. 64, pp. 2153-2209, Dec. 1985.

[3] L. G. Kazovsky, "Impact of laser phase noise on optical heterodyne communications systems," J. Opt. Commun., vol. 7, pp. 66-77, June 1986.

[4] G. J. Foschini, L. J. Greenstein, and G. Vannucci, "Noncoherent detection of coherent lightwave signals corrupted by phase noise," IEEE Trans. Commun., vol. COM36, pp. 306-314, Mar. 1988.

[5] G. J. Foschini and G. Vannucci, "Characterizing filtered light waves corrupted by phase noise," IEEE Trans. Inform. Theory, vol. IT-34, pp. 1437-1448, Nov. 1988.

[6] G. J. Foschini, G. Vannucci, and L. J. Greenstein, "Envelope statistics for filtered optical signals corrupted by phase noise," IEEE Trans. Commun., vol. COM-37, pp. 1293-1302, Dec. 1989.

[7] I. Garrett and G. Jacobsen, "Phase noise in weakly coherent systems," IEE Proc., vol. 136, pp. 159-165, June 1989.

[8] I. Garrett, D. J. Bond, J. B. Waite, D. S. L. Lettis, and G. Jacobsen, "Impact of phase noise in weakly coherent systems: A new and accurate approach," J. Lightwave Technol., vol. 8, pp. 329-337, Mar. 1990.

[9] L. G. Kazovsky and O. K. Tonguz, "ASK and FSK coherent lightwave systems: A simplified approximate analysis," J. Lightwave Technol., vol. 8, pp. 338-352, Mar. 1990. 
[10] Y. Bykovskii et al., "Use of a Fabry-Perot resonator for the stabilization of the frequency of an injection laser," Soviet Phys.-Semicond., vol. 4, pp. 580-583, 1970.

[11] F. Favre and D. L. Guen, "High frequency stability of laser diode for heterodyne communication systems," Electron. Lett., vol. 16, no. 18, pp. 709-710, 1980.

[12] A. Dandridge and A. B. Tveten, "Electronic phase-noise suppression in diode lasers," Electron. Lett., vol. 17, pp. 937-938, Dec. 1981.

[13] B. Glance, "Performance of AFC for phase noise reduction of optical sources," Electron. Lett., vol. 21, pp. 994-996, Oct. 1987.

[14] M. Ohtsu and S. Kotajima, "Linewidth reduction of a semiconductor laser by electrical feedback," IEEE J. Quantum Electron., vol. QE-21, pp. 1905-1912, Dec. 1985.

[15] E. A. Swanson and S. B. Alexander, "Wide bandwidth frequency noise suppression and FM equalization of semiconductor lasers," in Proceedings of Cleo 91, (Baltimore, Md), pp. CPDP37-1, 1991.

[16] A. Yariv, Optical Electronics. Holt, Rinehart and Winston, 1985.

[17] P. Humblet, "Design and performances of optical matched filters," in Proc. Globecom '91, (Phoenix, AZ), Dec. 1991.

[18] L. G. Kazovsky, P. Meissner, and E. Patzak, "ASK multiport optical homodyne systems," J. Lightwave Technol., vol. 5, pp. 770-791, June 1987.

[19] M. Azizoglu and P. A. Humblet, "Envelope detection of orthogonal signals with phase noise," J. Lightwave Technol., vol. 9, pp. 1398-1410, Oct. 1991.

[20] H. L. Van Trees, Detection, Estimation, and Modulation Theory, Part I. New York: John Wiley \& Sons, 1968. 
[21] J. G. Proakis, Digital Communications. New York: McGraw-Hill, 1983. 


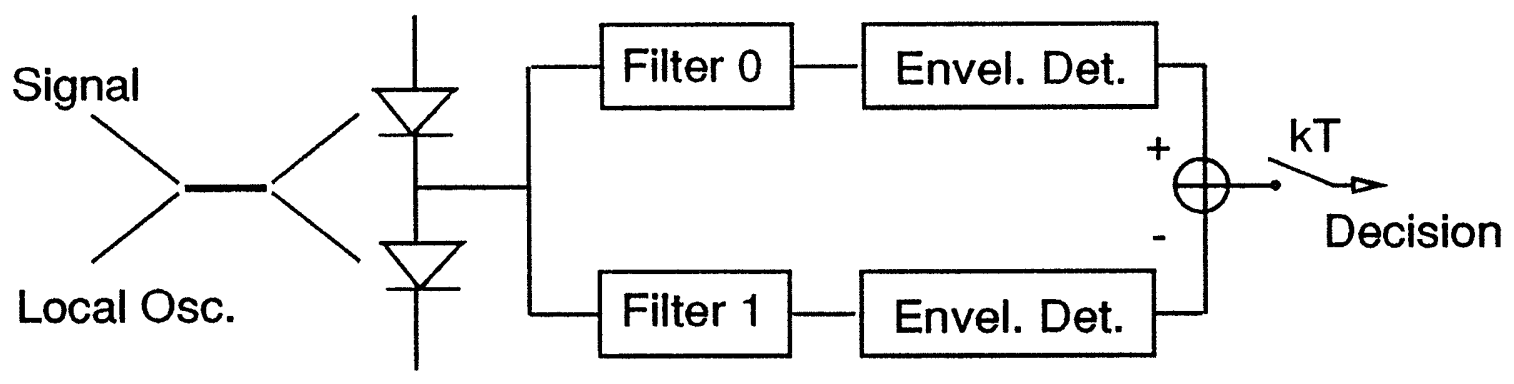

Figure 1: Structure of FSK Heterodyne Envelope Receiver

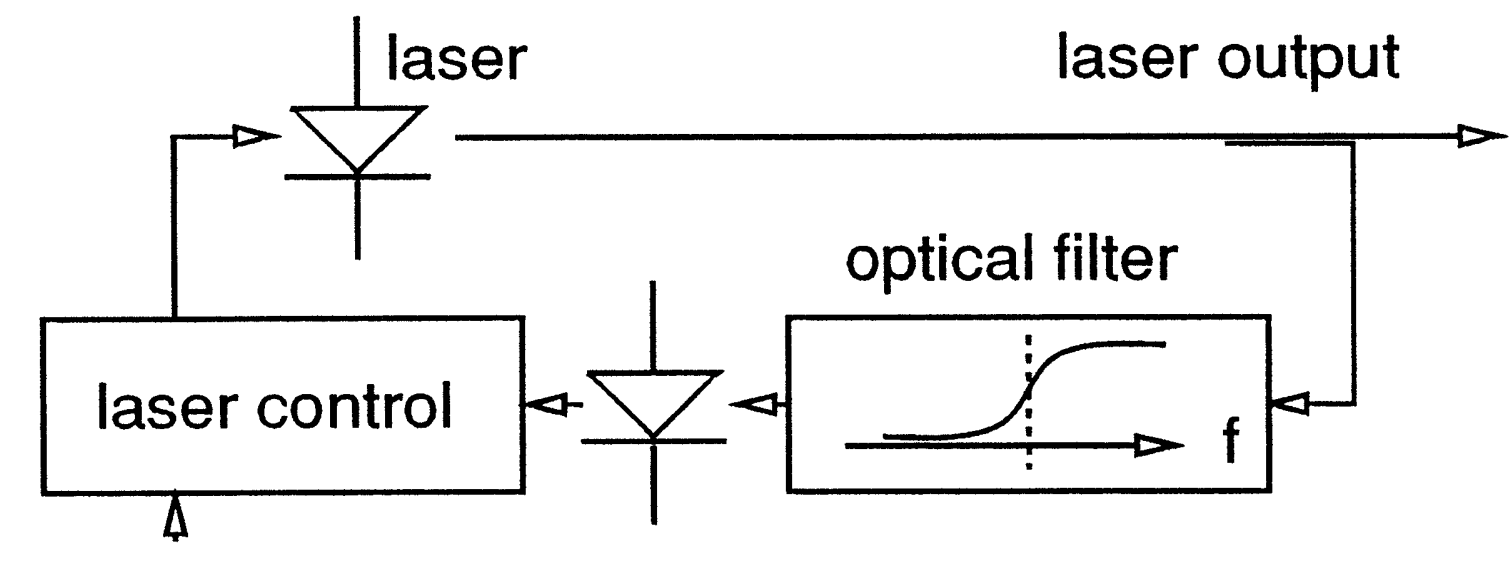

modulation photodetector

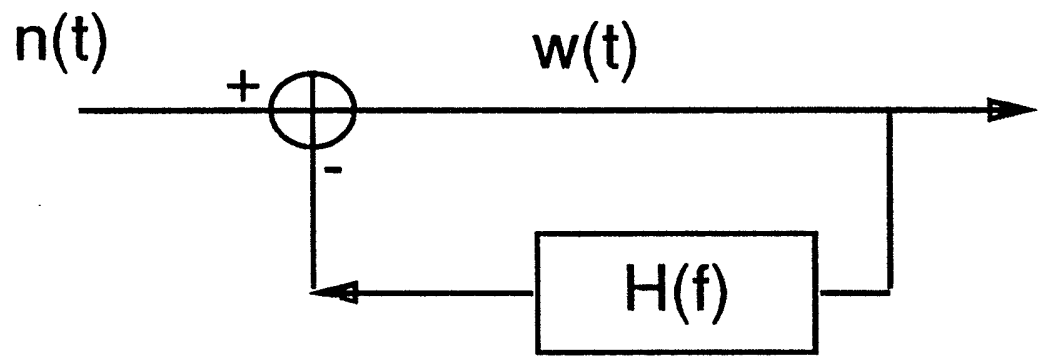

Figure 2: Frequency control system and model 


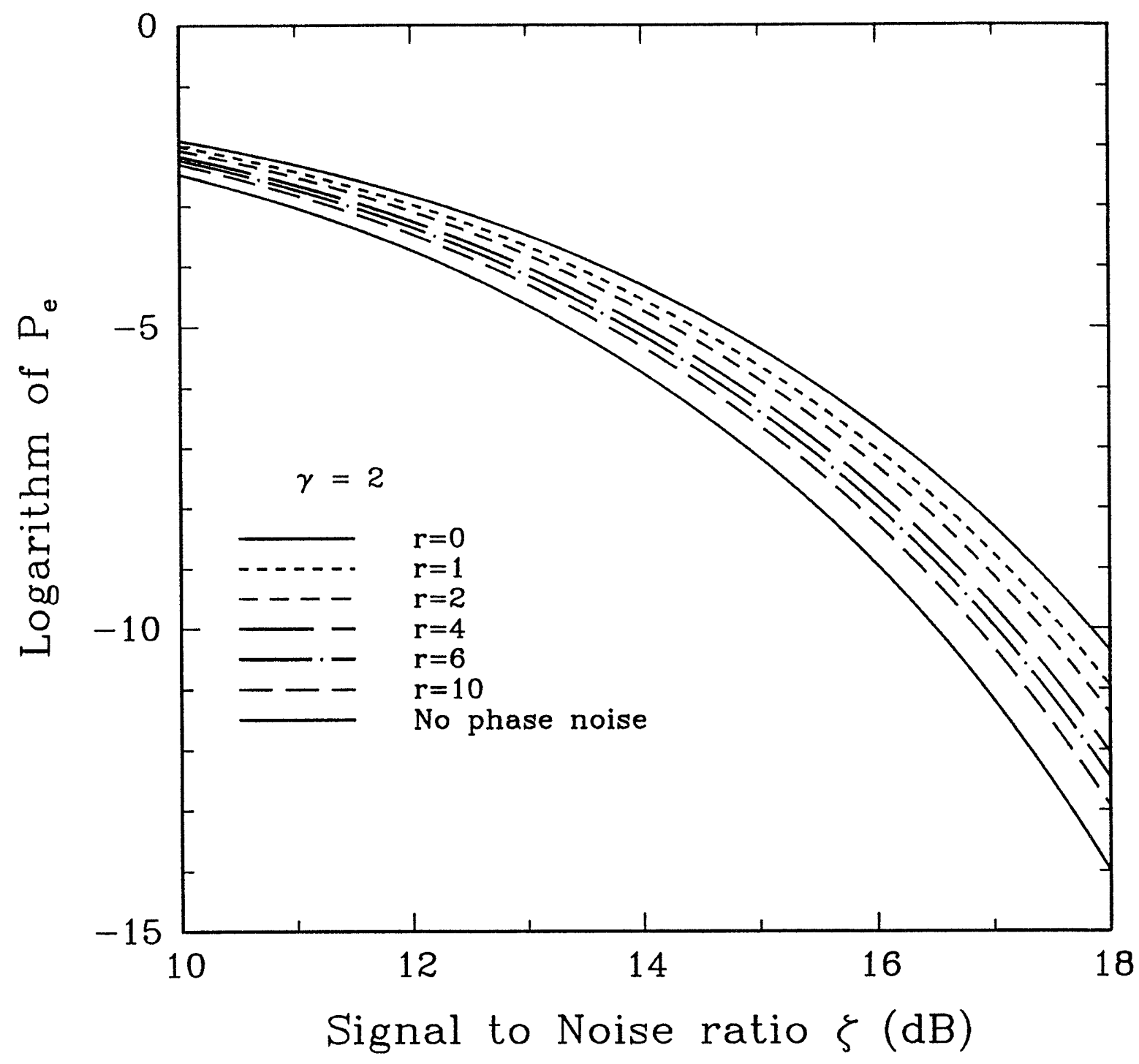

Figure 3: Lowerbound on the probability of error for $\gamma=2$ 


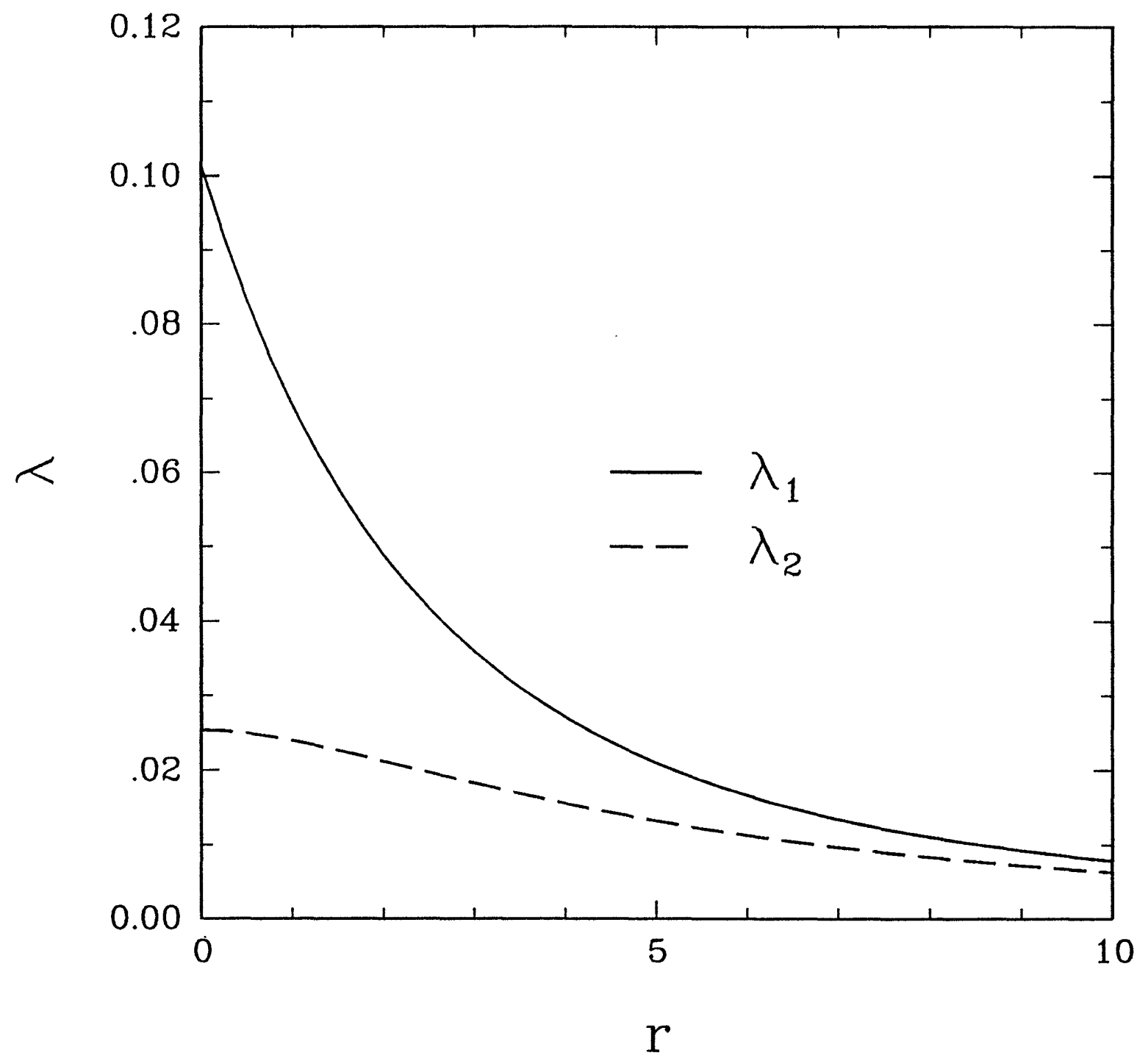

Figure 4: First two eigenvalues as functions of $r$ 


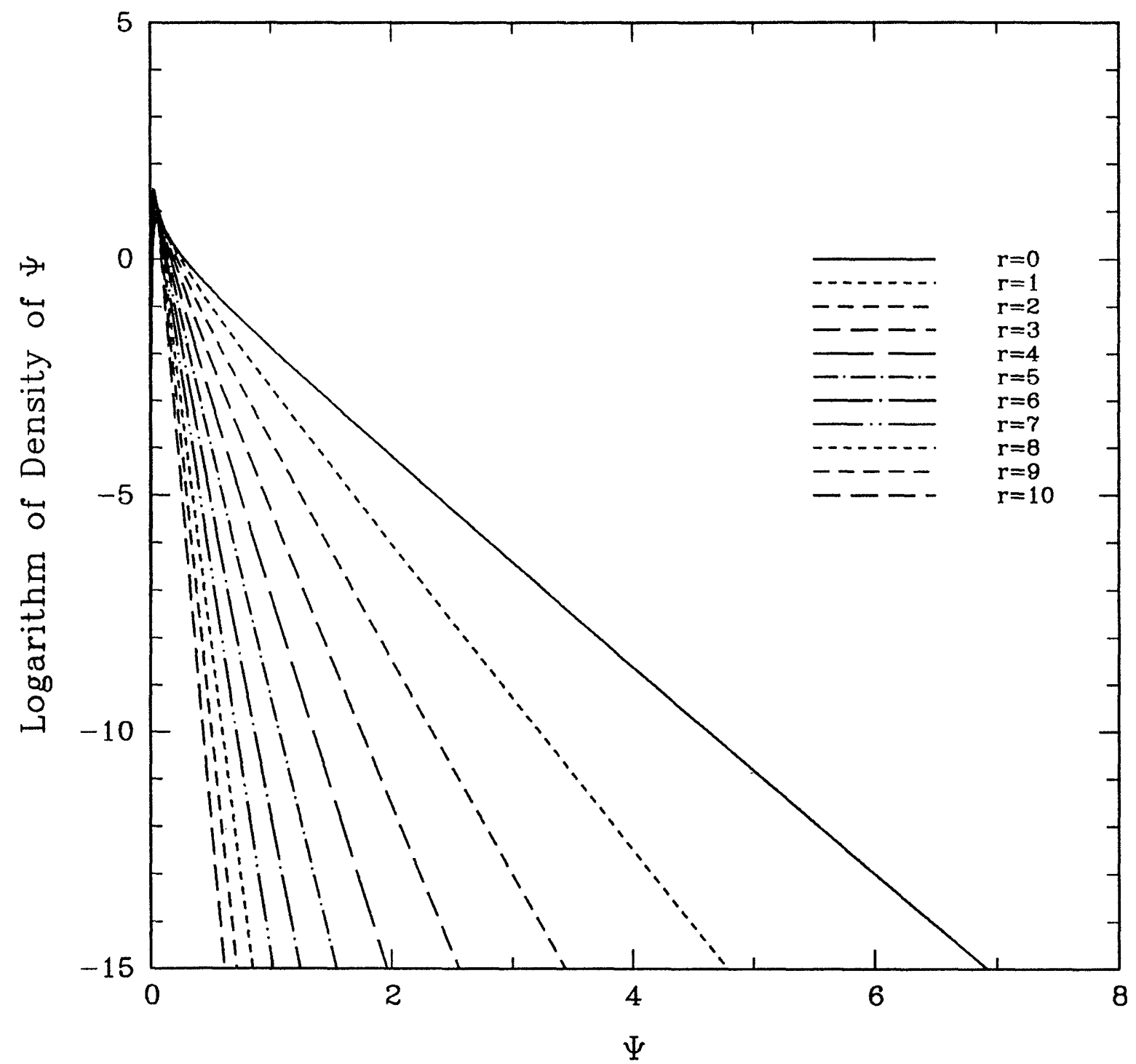

Figure 5: Density of $\Psi$ for various values of $r$ 


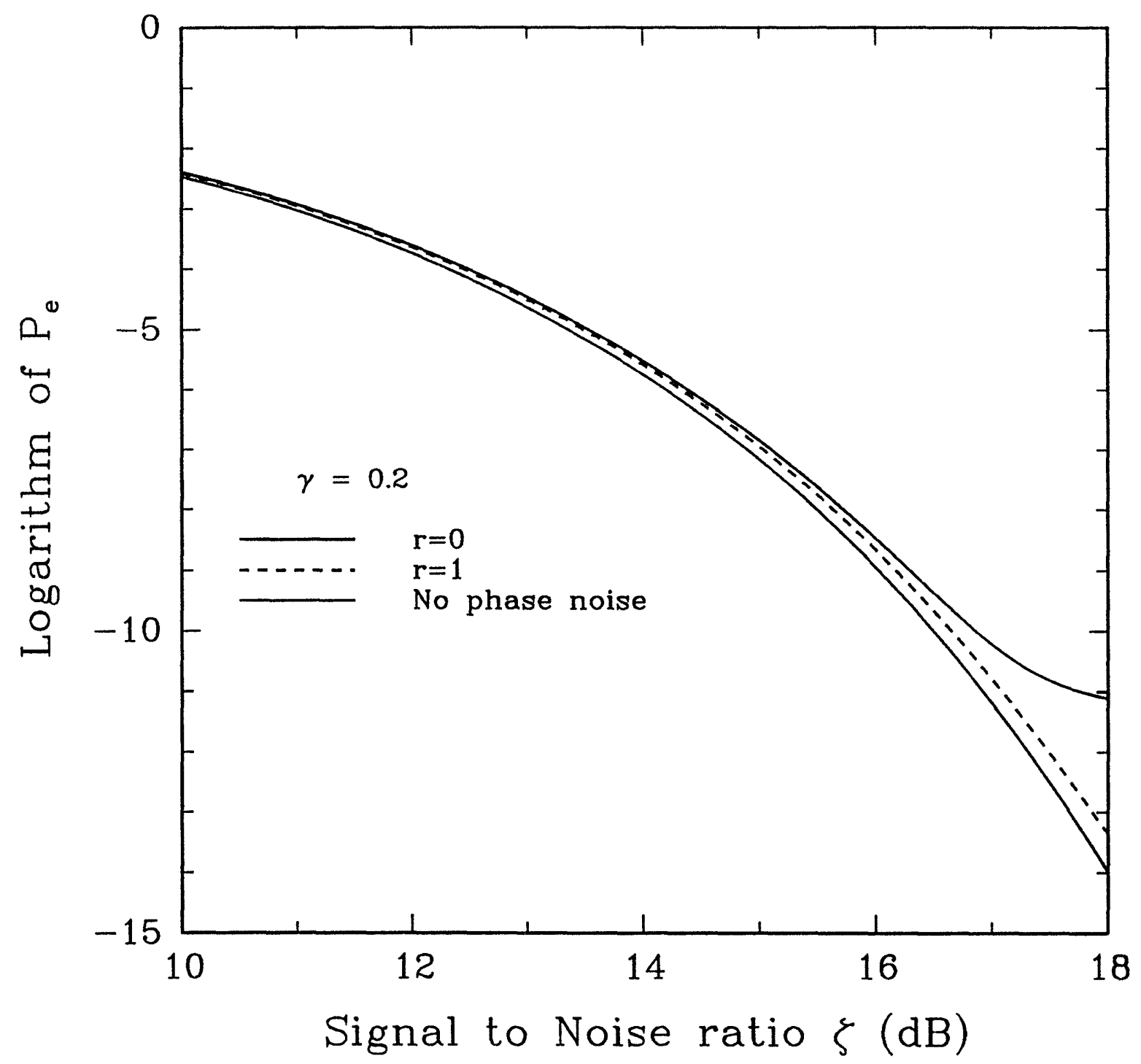

Figure 6: Upperbound on the probability of error for $\gamma=.2$ 


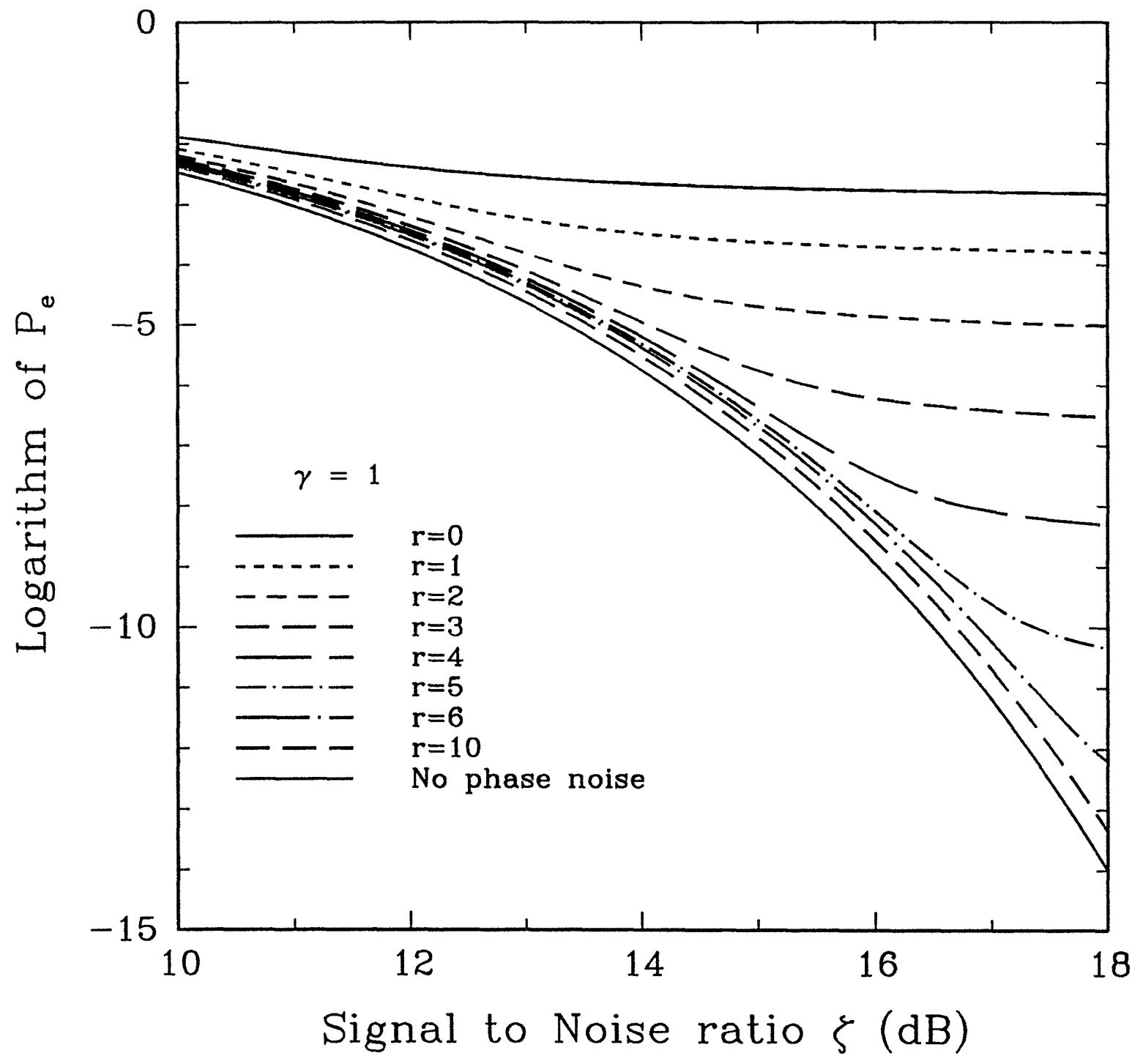

Figure 7: Upperbound on the probability of error for $\gamma=1$ 


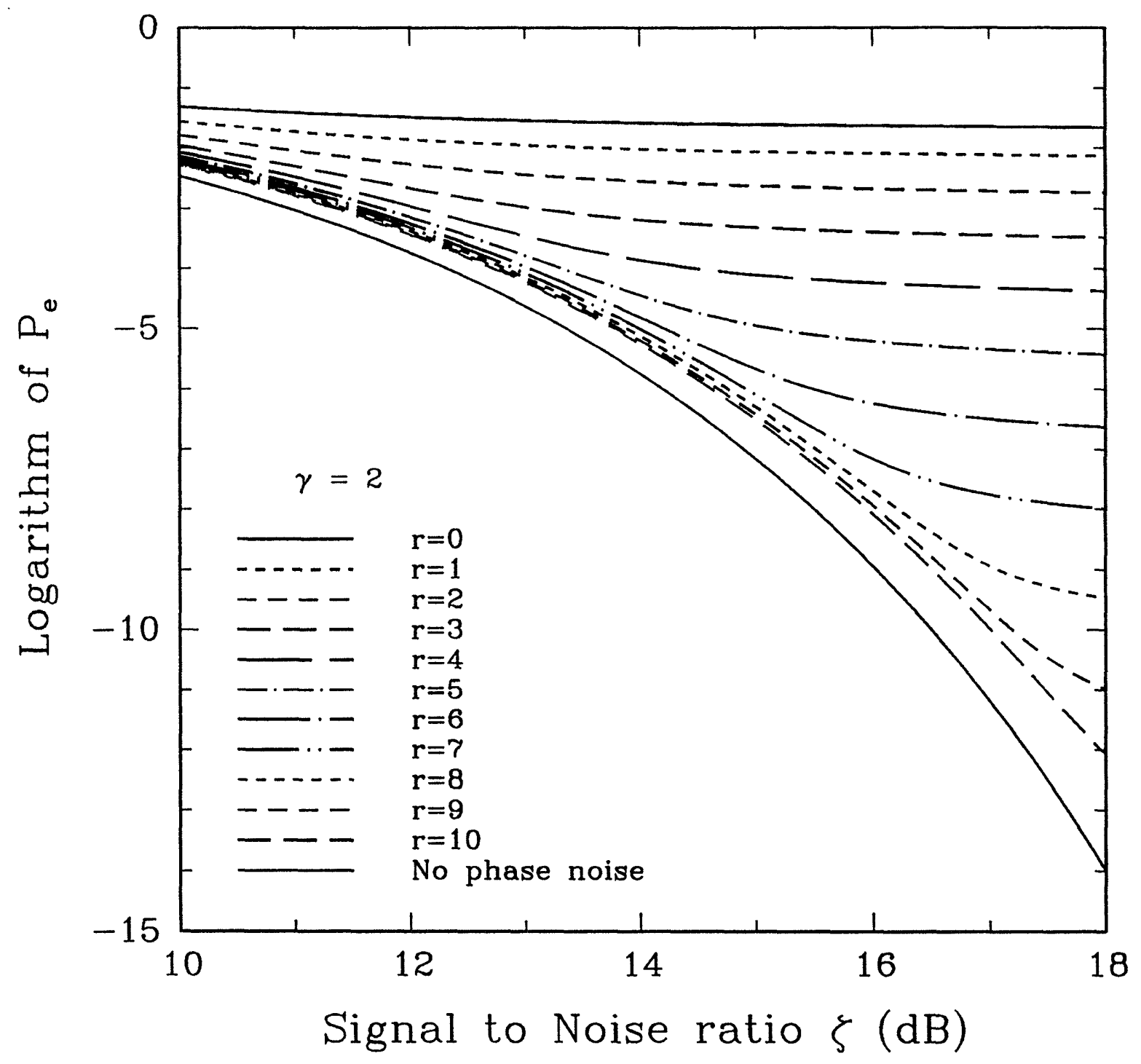

Figure 8: Upperbound on the probability of error for $\gamma=2$ 


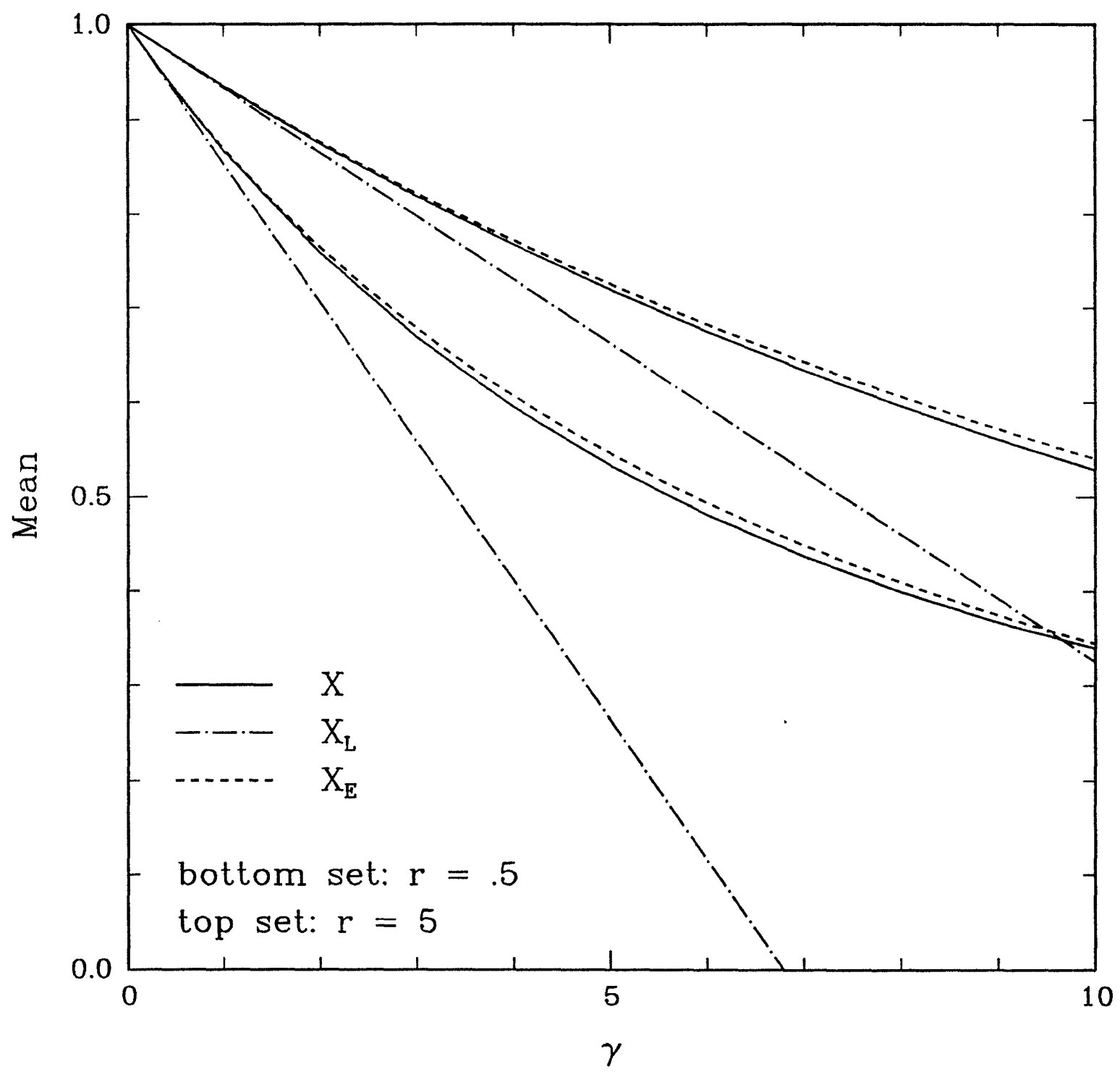

Figure 9: Comparison of $\bar{X}, \overline{X_{L}}$ and $\overline{X_{E}}$ as functions of $\gamma$ 


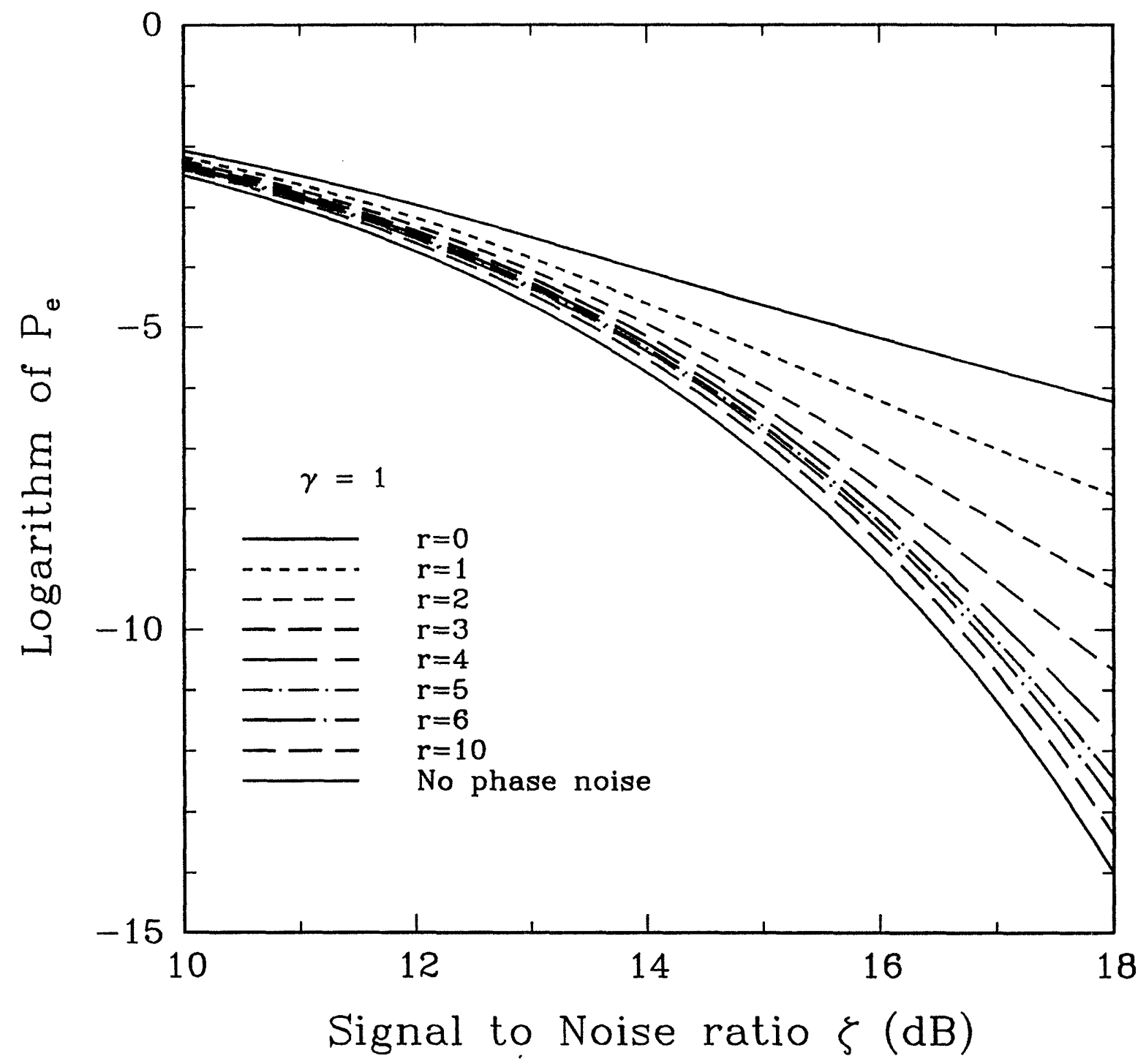

Figure 10: Probability of error predicted by the exponential approximation for $\gamma=1$ 


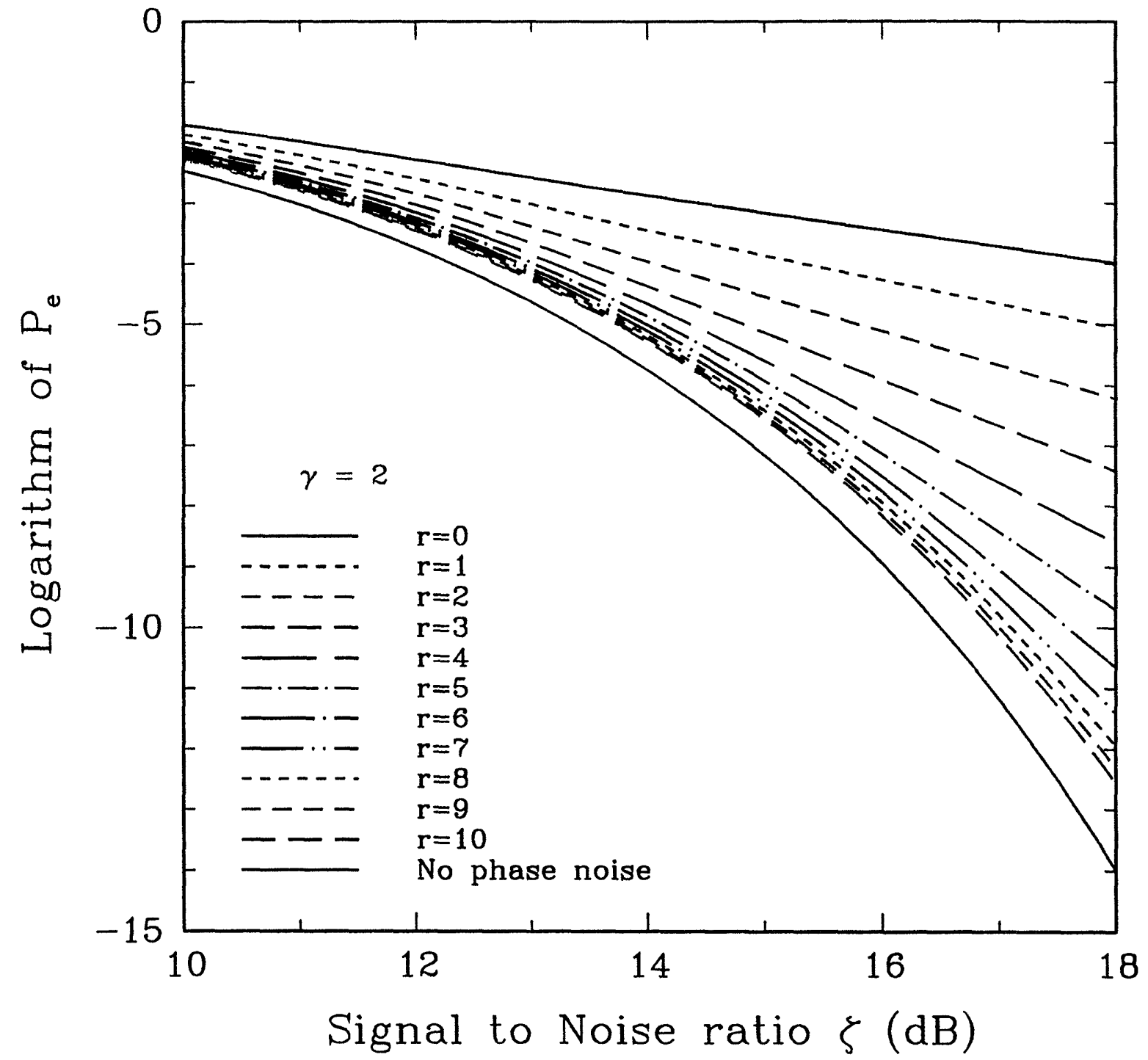

Figure 11: Probability of error predicted by the exponential approximation for $\gamma=2$ 


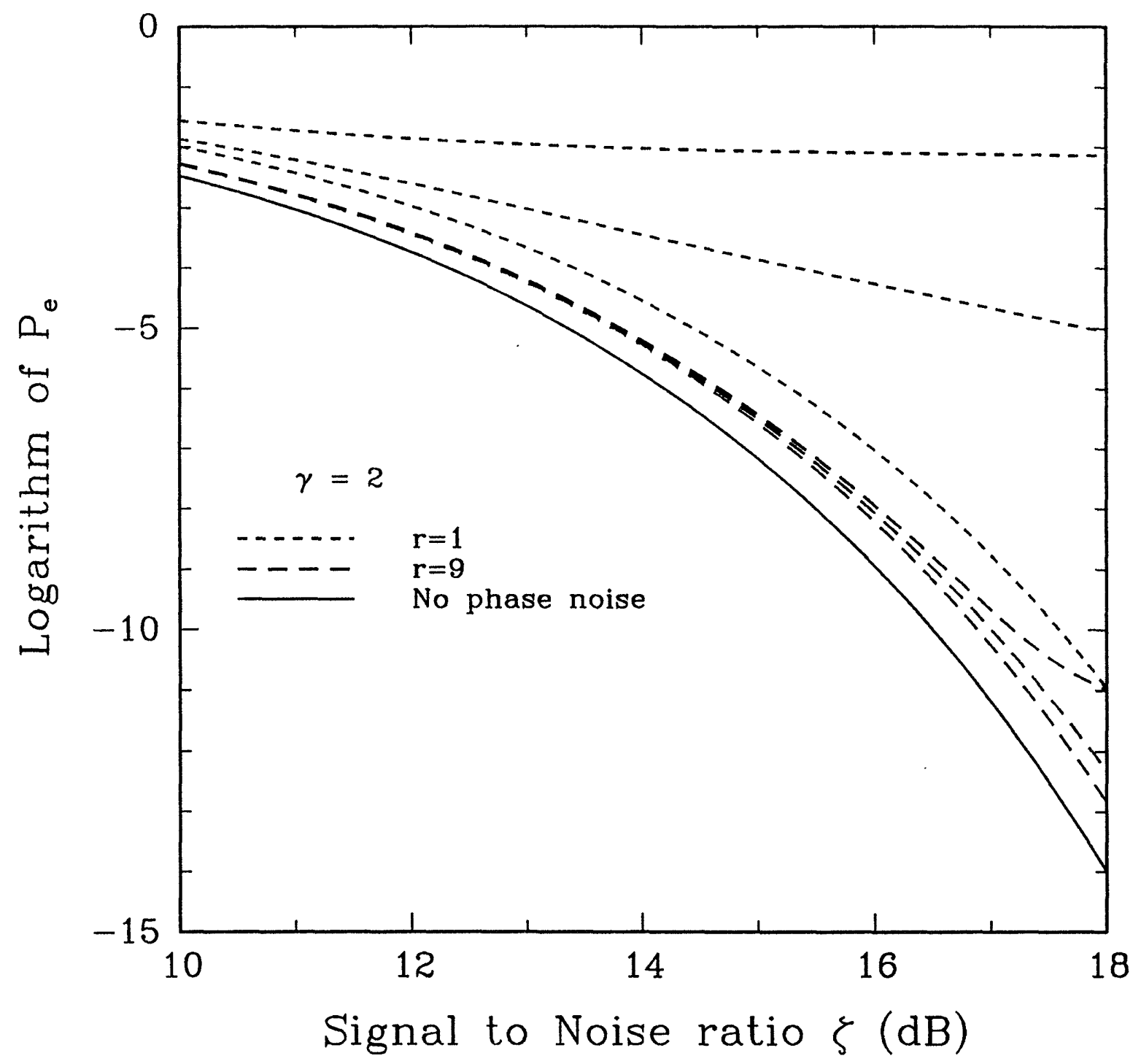

Figure 12: Comparison of upper bound, lower bound and approximation to the error probability 